\title{
Modal Structure of Integrated Semiconductor Ring Lasers with Output Waveguides
}

\author{
A. Pérez-Serrano ${ }^{1}$, S. Fürst ${ }^{2}$, A. Scirè ${ }^{1}$, J. Javaloyes ${ }^{3}$, S. Balle ${ }^{3}$ and M. Sorel ${ }^{2}$ \\ ${ }^{1}$ Instituto de Física Interdisciplinar y Sistemas Complejos, IFISC (CSIC-UIB) \\ Campus UIB, Ctra. Valldemossa km. 7.5, E-07122 Palma de Mallorca, Spain. \\ 2 Dept. of Electronics and Electrical Engineering, University of Glasgow, \\ Oakfield Avenue, Glasgow G12 8LT, United Kingdom. \\ ${ }^{3}$ Instituto Mediterráneo de Estudios Avanzados, IMEDEA (CSIC-UIB) \\ C/ Miquel Marqués 21, E-07190 Esporles, Spain.
}

\begin{abstract}
We have experimentally and theoretically analyzed the modal properties of semiconductor ring lasers and the wavelength jumps that occur in connection with directional switching above threshold.

Index Terms-Semiconductor lasers, ring lasers, modal prop-
\end{abstract} erties, coupled cavities, laser dynamics

\section{INTRODUCTION}

Semiconductor Ring Lasers (SRLs) are technologically appealing devices for the fabrication of micro-cavity lasers and densely integrated photonics circuits. The bistability of the clockwise $(\mathrm{CW})$ and counterclockwise $(\mathrm{CCW})$ states opens up a new scenario in the development of all-optical memories and signal processing [1], [2] owing to the fast directional switching among these states.Besides their switching characteristics, SRLs show several unexpected dynamical behaviors - such as hysteresis in the lasing direction [3], [4] — and lasing mode selection rules that are quite different from those of FabryPerot lasers [5]. The understanding of these phenomena and their influence over bistability is necessary for the development of practical devices based on SRLs.

In order to investigate the large mode-hop exhibited by SRLs for increasing currents, we precisely measured the passive transfer function of the devices in the frequency domain, together with its emission wavelength when biased above threshold. The transfer function can be theoretically explained by considering the perturbation induced by the couplers and the output waveguides, which induces a symmetry breaking in the resonant cavity and a modulation of the cavity losses. For the geometry considered, the cavity losses have a wavelength periodicity that corresponds to three ring cavity modes, which explains the measured hops in wavelength as the bias current of the laser is increased.

\section{Device Characterization}

Our SRL was fabricated at U. Glasgow as described in [6], with ring-cavity radius of $300 \mu \mathrm{m}$. Light from the SRL is extracted through two couplers with a theoretical coupling ratio of $12 \%$ into two output waveguides tilted $10^{\circ}$ to the cleaved facets to minimize backreflections. The lower waveguide arms — ports \#3 (left) and \# 4 (right) — are reversed

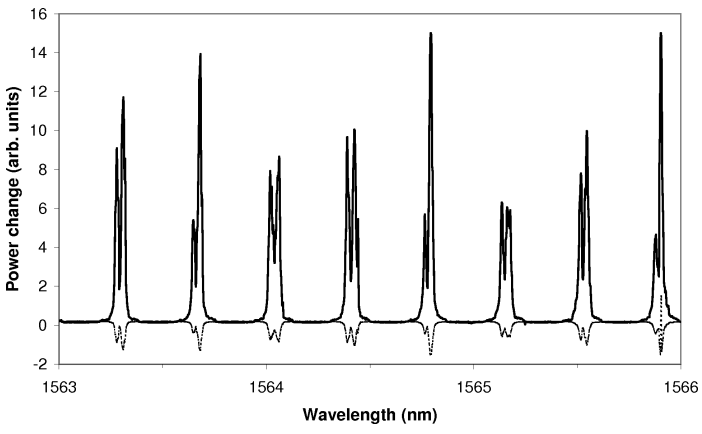

Fig. 1. Experimental resonances.

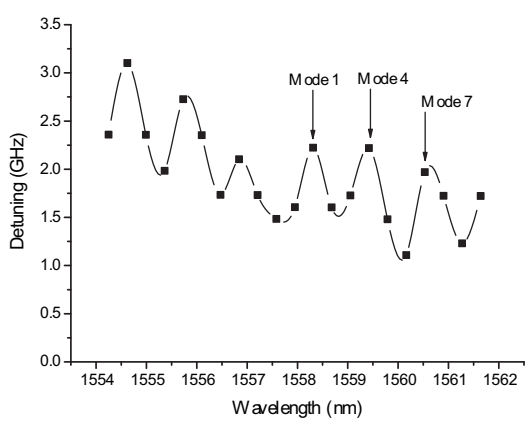

Fig. 2. Measured detuning between doublets.

biased and used as integrated photodetectors, while the left facet of the upper waveguide (port \#1) is used to inject an external optical field if required.

In order to analyze the cavity resonances of the SRL, we inject through port \#1 a monochromatic field from a tunable laser and we measure the photo-current generated in ports \#3 and \#4 vs the wavelength of the injected field (see Fig. 1).

For a current slightly below the SRL threshold (approx. 32.5 $\mathrm{mA}$ ), the power variation at port \# 3 shows a series of upward doublets with a major spacing of $0.4 \mathrm{~nm}$ and a doublet splitting of about $4 \mathrm{GHz}$. The power variation at port \# 4 displays the same structure in the downward direction, i. e., as dips on the noise level. The depth of the dips is strongly dependent on the current applied to the SRL, and the dips disappear 
for currents below $30 \mathrm{~mA}$. The doublet splitting varies with wavelength (see Fig. 2) and it is modulated at roughly one third of the mode spacing. Fig. 3 shows the LI curve of the SRL measured at ports \# 3 and \# 4 (with no injected field). Close to threshold ( approx. 29.0 mA) the SRL emits bidirectionally, but it rapidly changes to (almost) unidirectional emission. In this regime, further increases of the current lead to directional reversal which is accompanied by abrupt wavelength jumps by three mode spacings. In between these jumps, the emission wavelength remains constant except for the small thermal drift due to Joule heating. At high currents, bidirectional although very unstable emission is recovered.

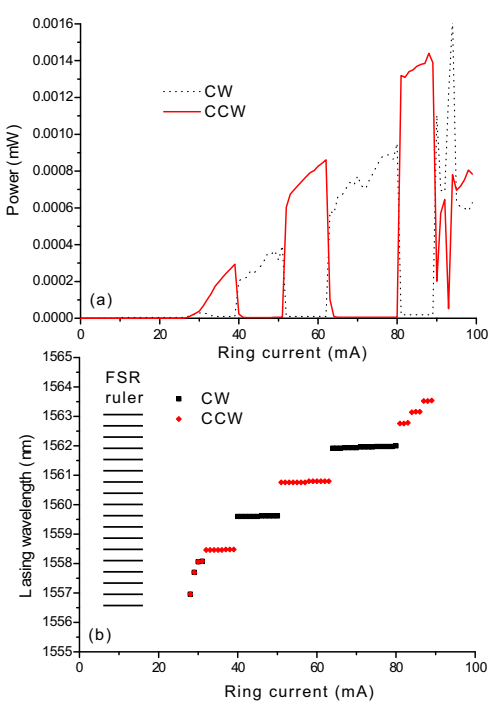

Fig. 3. Experimental L-I curve showing the wavelength jumps.

\section{THEORETICAL ANALYSIS}

In order to explain the injection experiments below threshold, we compute the transfer matrix of the complete SRL structure [7] assuming that the two couplers are identical, lossless and with a residual reflectivity due to their point-like character. Small reflectivity from output facets \#1 and \# 2 is included, but not from \#3 or \#4 since the corresponding output waveguides are reverse biased. The theoretical results for the power at port \# 3 are in good agreement with the results shown in Fig. 1, the section lengths have been taken from the device layout and facet reflectivities have been adjusted to match the experiments. In order to resolve the doublets and have a good agreement with the experiment, a small amount of gain in the SRL has to be included. The transfer matrix analysis does not reproduce the observations at port \# 4 because they correspond to the suppression of amplified spontaneous emission in the SRL cavity, which is not included in the model.

On the other hand, the roundtrip condition for the SRL modes in a resonator with equal arms of length $L / 2$ can be formulated as

$$
e^{2 i q L}-a e^{i q L}+b=0,
$$

where $q$ is the propagation constant. In (1), $b=\left(r_{u} r_{u}^{\prime}-\right.$ $\left.t_{u} t_{u}^{\prime}\right)^{-1}\left(r_{d} r_{d}^{\prime}-t_{d} t_{d}^{\prime}\right)^{-1}, a=\left(r_{u} r_{d}+r_{u}^{\prime} r_{d}^{\prime}+t_{u}^{\prime} t_{d}+t_{u} t_{d}^{\prime}\right) b$ and $t_{u(l)}$ and $r_{u(l)}$ denote the wavelength-dependent transmittivity and reflectivity of the upper (lower) coupler for $\mathrm{CW}$ waves, while primed symbols denote the same magnitudes for $\mathrm{CCW}$ waves.

Thus, the SRL modes are given by

$q_{m}^{ \pm} L=2 \pi m-i \ln \left[\frac{a}{2} \pm \sqrt{\left(\frac{a}{2}\right)^{2}-b}\right] \equiv 2 \pi m-i \ln Q_{ \pm}$.

The two branches of solutions correspond to the experimentally observed doublets, and their splitting normalized to the free-spectral range of the SRL is thus given by

$$
\Delta=\frac{c}{2 \pi n}\left\{\operatorname{Im}\left[\ln \left(\frac{Q_{-}}{Q_{+}}\right)\right]-\alpha \operatorname{Re}\left[\ln \left(\frac{Q_{-}}{Q_{+}}\right)\right]\right\},
$$

$\alpha$ standing for the linewidth enhancement factor in the SRL. The results for the detuning are shown in Fig. 4, and are in good agreement with the observations (Fig. 2).

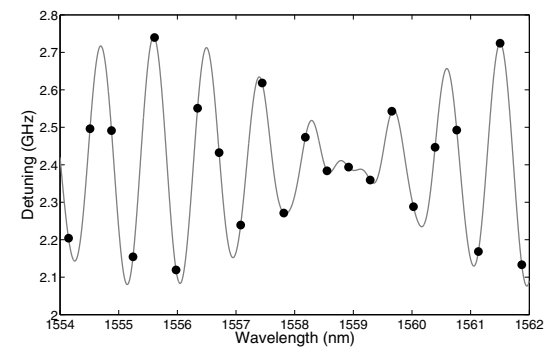

Fig. 4. Theoretical detuning between doublets.

\section{ACKNOWLEDGMENT}

This work was funded by the European project IOLOS IST2005-34743, Spanish project PhoDeCC TEC2006-10009/MIC and Balear Government project QULMI PROGECIB-5A. A.S. acknowledges Ramón y Cajal program by Spanish MEC. S.B. and J.J. acknowledge financial support from project TEC200613887-C05-03. J.J. acknowledges support from the Juan de La Cierva program by the Spanish MEC.

\section{REFERENCES}

[1] V.R. Almeida, C.A. Barrios, R.R. Panepucco, M. Lipson, M.A. Foster, D.G. Ouzounov and A.L. Gaeta, "All-optical switching on a silicon chip", Opt. Lett. 29, 2867 (2004).

[2] M. T. Hill, H. J. S. Dorren, T. de Vrie, X. J. M. Leijtens, J. H. den Besten, B. Smalbrugge, Y. S. Oei, H. Binsma, G. D. Khoe and M. K. Smit, "A fast low-power optical memory based on coupled micro-ring laser", Nature 432, 206 (2004).

[3] M.F. Booth, A. Schremer and J.M. Ballantyne, "Spatial beam switching and bistability in a diode ring laser", Appl. Phys. Lett. 76, 1095 (2000).

[4] M. Sorel, P. J. R. Laybourn, G. Giuliani and S. Donati, "Unidirectional bistability in semiconductor waveguide ring lasers", Appl. Phys. Lett. 80, 3051 (2002).

[5] M. Sorel, G. Giuliani, A. Scirè, R. Miglierina, S. Donati and P.J.R. Laybourn, IEEE J. Quantum Electron. 39, 1187 (2003).

[6] S. Furst and M. Sorel, "Cavity-enhanced four-wave mixing in semiconductor ring lasers", IEEE Photon. Tech. Lett. 20(5), 366 (2008).

[7] A.E. Siegman, Lasers, University Science Books, Mill Valley, CA, pp. 398-426 (1986). 